\title{
Interaction of Supermassive Black Holes with their Stellar and Dark Matter Environments
}

\author{
David Merritt \\ Rochester Institute of Technology, Rochester, NY, USA
}

\section{Introduction}

While supermassive black holes probably gained most of their mass via accretion of gas, the galactic nuclei in which they are currently situated are dominated by stars. This article reviews recent theoretical work on the interaction between black holes and their stellar environment, and highlights ways in which the observed structure of galactic nuclei can be used to constrain the formation history of black holes. Nuclei may also contain dark matter, and the possibility of detecting supersymmetric particles via annihilation radiation from the Galactic center has generated some interest 26]. The evolution of the dark matter distribution in the presence of a black hole in a stellar nucleus is also discussed.

\section{Matter Distribution around Black Holes}

Figure 1shows two natural models for the evolution of the stellar density around a black hole. If the star-star relaxation time is long, the form of $\rho(r)$ will still reflect the way the nucleus formed. The simplest possible picture is growth of a black hole at a fixed location in space due to some spherically-symmetric accretion process. As the black hole grows it pulls in stars. If the growth time is long ("adiabatic") compared with orbital periods, the change in $\rho(r)$ is unique and straightforward to compute 5164. Figure 10 shows the result if the initial density is a power law, $\rho \propto r^{-\gamma_{0}}$. The final profile is well described as two power laws joined at a radius $r_{\text {cusp }}$ where

$$
r_{\text {cusp }}=\alpha r_{h}, \quad 0.19 \lesssim \alpha \lesssim 0.22, \quad 0.5 \leq \gamma_{0} \leq 1.5
$$

and $r_{h}$ is the radius containing a mass in stars equal to twice the final black hole mass. The final density within $r_{\text {cusp }}$ is

$$
\rho \propto r^{-\gamma}, \quad 2.29 \leq \gamma \leq 2.5
$$

This result is insensitive to the geometry (flattened, triaxial, etc.) and degree of rotation of the nucleus.

If the nucleus is older than roughly one relaxation time, a different sort of mass distribution is set up around the black hole, corresponding to a steadystate solution of the Fokker-Planck equation. This steady state has a nearly zero net flux of stars with respect to energy and the density profile is given 


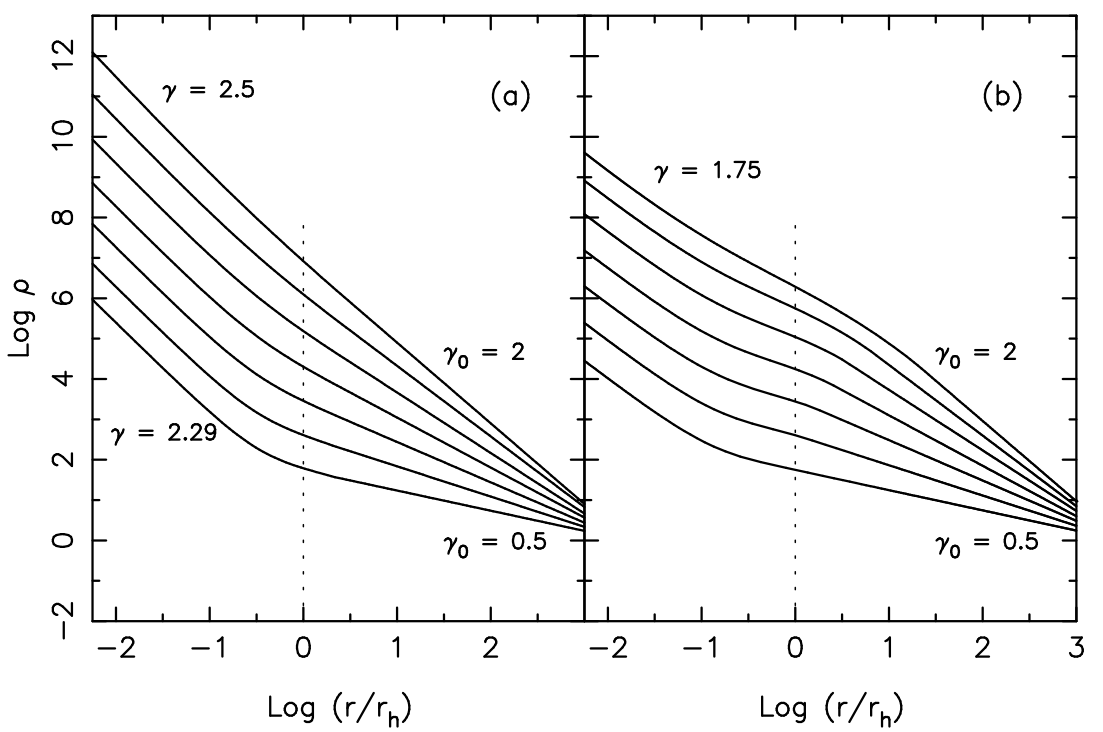

Fig. 1. (a) "Collisionless" density profiles, resulting from growth of a black hole at a fixed location. Initial profiles were power laws, $\rho \propto r^{-\gamma_{0}}$, with $\gamma_{0}$ increasing upwards in steps of 0.25 . The radial scale is normalized to $r_{h}$ as defined in the initial (pre-blackhole) galaxy. (b) "Collisional" density profiles, computed as steady-state solutions to the Fokker-Planck equation. The density inside $\sim 0.1 r_{h}$ satisfies $\rho \propto r^{-7 / 4}$.

uniquely as $\rho \propto r^{-7 / 4}$ near the black hole 573 . Figure 1b shows the result, again assuming various power-law slopes for the initial $\rho(r)$. The $r^{-7 / 4}$ dependence is only reached at $r \lesssim r_{\text {cusp }} \approx 0.1 r_{h}$.

The condition for the appearance of a collisional, $\gamma=7 / 4$ cusp is that the relaxation time,

$$
T_{r}(r)=\frac{\sqrt{2} \sigma(r)^{3}}{\pi G^{2} m \rho(r) \log \Lambda},
$$

measured at $\sim r_{h}$ be less than the age of the nucleus 352. Figure 4 shows $T_{r}\left(r_{h}\right)$ for a sample of elliptical galaxies drawn from Faber et al. [11. Only a few galaxies, all low luminosity ellipticals, have $T_{r}<10^{10} \mathrm{yr}$. Two examples are well known: the Milky Way bulge, which has $T_{r} \approx 2 \times 10^{9}$ yr (assuming $m=M_{\odot}$ ), and M32, for which $T_{r} \approx 3 \times 10^{9}$ yr. Both galaxies should have collisionally relaxed nuclei. In the Milky Way, $r_{h} \approx 1.7 \mathrm{pc} \approx 40^{\prime \prime}\left[15\right.$ and $r_{\text {cusp }} \approx 4^{\prime \prime}$. The stellar density profile has been determined into $\sim 0.1^{\prime \prime}$; it is a broken power law with $\gamma \approx 1.4$ inside of $\sim 10^{\prime \prime}$ and $\gamma \approx 2.0$ outside 15 . The break radius is reasonably close to the predicted value of $r_{\text {cusp }}$ but the interior slope is significantly flatter than $7 / 4$. In the case of M32, $r_{h} \approx 2.9 \mathrm{pc} \approx 0.9^{\prime \prime}$ and $r_{\text {cusp }} \approx 0.1^{\prime \prime} 28$. The stellar luminosity density has been determined into only $\sim 0.15^{\prime \prime}$, not quite far enough to test for the existence of a collisional cusp. The situation is worse for more distant galaxies that might harbor collisionally relaxed nuclei, making the 


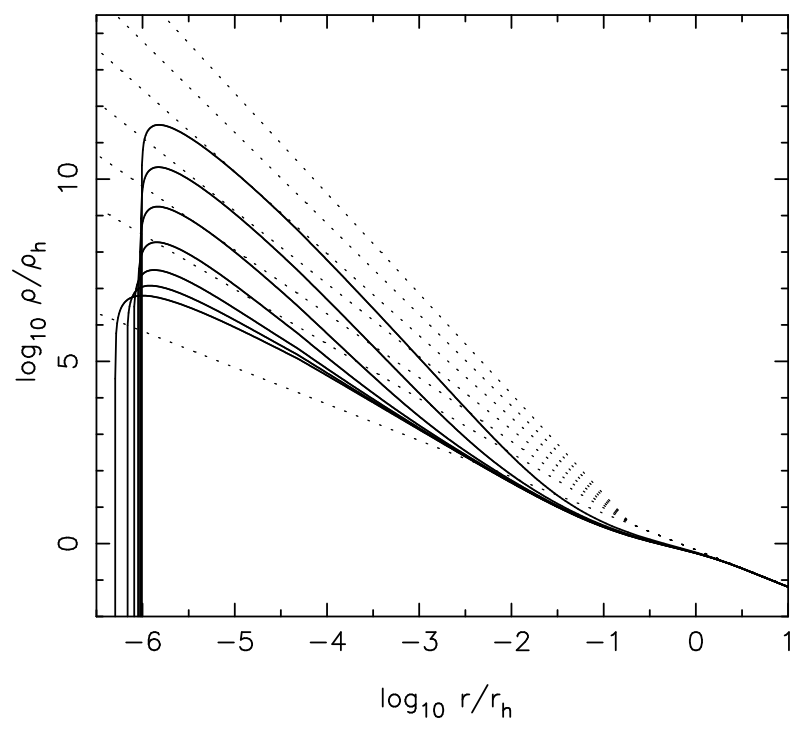

Fig. 2. Dark matter density at $t=0$ (dotted lines) and $10^{10} \mathrm{yr}$ (solid lines) after heating by stars in the Galactic bulge 38.

Milky Way nucleus the only system in which the theory can currently be tested. The fact that $\gamma<7 / 4$ in the Milky Way nucleus may be due to mass segregation which produces a flatter density profile for the less-massive stars [4 50].

Particle dark matter, if present in galactic nuclei, would respond to the growth of the black hole according to the "collisionless" model. Its density near the black hole could therefore be very high, resulting in a substantial rate of self-annihilations if the particles are supersymmetric neutralinos [17. The dark matter distribution would also evolve due to scattering off of stars, on a time scale close to $T_{r}$, the star-star relaxation time 273816]. In effect, the stars act as a heat source for the dark matter. Figure 2 illustrates this via time-dependent solutions to the Fokker-Planck equation. Given the appropriate normalization with respect to the dark matter density near the solar circle, this nuclear profile implies a potentially observable flux of annihilation products 38.

\section{Feeding Rates}

In a spherical galaxy, stars or dark matter particles with angular momenta less than $J_{l c} \approx \sqrt{2 G M_{\bullet} r_{t}}$ will pass within a distance $r_{t}$ of the center of the black hole. The "loss cone" is that set of orbits satisfying $J \leq J_{l c}(E)$. If we imagine that the black hole is embedded in a steady-state galaxy populated by stars with $J \leq J_{l c}$, stars on loss cone orbits will be consumed in one orbital period or less, giving a transitory feeding rate of $\sim 4 \pi^{2} f(E, 0) J_{l c}^{2}(E)$ stars per unit energy per unit time. This is sometimes called the "full loss cone" consumption rate and 


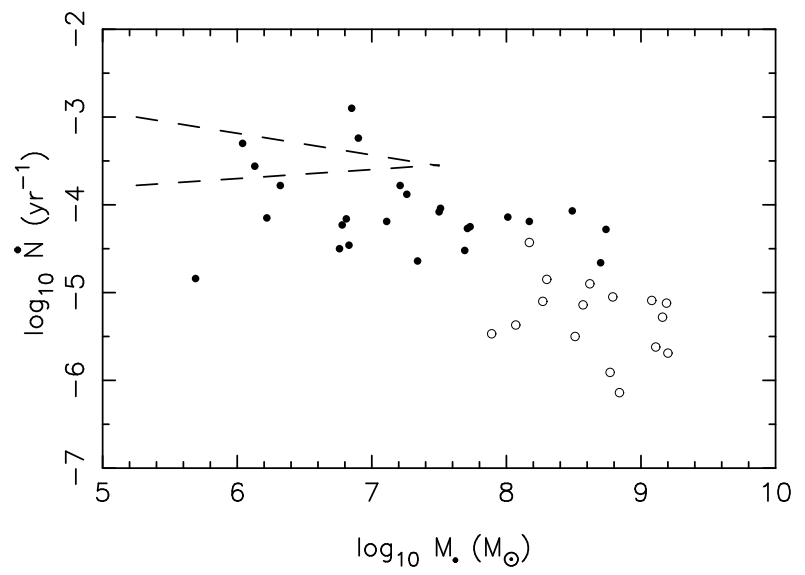

Fig. 3. Stellar tidal disruption rates in a sample of elliptical galaxies computed using the Cohn-Kulsrud [7] loss cone boundary conditions [60]. Open circles are "core" galaxies and filled circles are "power-law" galaxies. Upper and lower dashed lines are disruption rates in power-law nuclei with $\rho \propto r^{-2}$ and $r^{-1.5}$ respectively.

it is an effective upper limit to how quickly stars can be supplied to the black hole. For instance, in a $\rho \sim r^{-2}$ nucleus, the full loss cone feeding rate due to all stars with $E>\Phi\left(r_{h}\right)$ is $\sim \sigma^{5} r_{t} / G^{2} M_{\bullet}$ or $\sim \sigma^{5} / G c^{2}$ when $r_{t} \approx r_{s} \equiv 2 G M_{\bullet} / \sigma^{2}$. This is a high enough rate to grow black holes of the correct size after $10^{10} \mathrm{yr}$, i.e. $\sim 10^{-2} M_{\odot} \mathrm{yr}^{-1}$ for $\sigma \approx 200 \mathrm{~km} \mathrm{~s}^{-1}$.

Full loss cones have been postulated from time to time, perhaps first by Hills [22, later by P. Young and co-authors (for the "black-tide" model of quasar fueling) 6365, and most recently by Zhao, Haehnelt \& Rees 68, who noted that the $\sigma^{5}$ scaling is consistent with the $M_{\bullet}-\sigma$ relation. But while transient loss cone refilling during, say, galaxy mergers is imaginable, it is hard to see how a galaxy could arrange for the $10^{5}$ or more refilling events that would be needed to keep a loss cone continuously filled.

Instead, the loss cone is probably usually empty at radii $r<r_{\text {crit }}$, where $r_{c r i t}$ is the radius at which the scattering time into the loss cone equals the orbital period. Below $r_{\text {crit }}$, stars are lost the moment they enter the loss cone, while above $r_{\text {crit }}$, stars may scatter in and out of the loss cone in a single orbit. For $M_{\bullet} \approx 10^{7} M_{\odot}, r_{\text {crit }} \approx r_{h}$, and $r_{c r i t}<r_{h}$ at larger $M_{\bullet}$ [13. The feeding rate due to stars inside $r_{\text {crit }}$ is $\sim M\left(r_{\text {crit }}\right) / T_{r}\left(r_{\text {crit }}\right)$ since in one relaxation time a star's angular momentum can change by of order itself. Outside $r_{c r i t}$, the loss cone is effectively full but the feeding rate drops off rapidly with radius since the capture sphere subtends such a small angle (the "pinhole" regime). One finds in a careful calculation that the flux into the black hole is strongly peaked at energies near $\Phi\left(r_{\text {crit }}\right)$ 307.

Figure 3 shows tidal disruption rates computed in a sample of elliptical galaxies, with $r_{t}$ set to the tidal radius for solar-mass stars [60]. (For $M_{\bullet} \gtrsim 10^{8} M_{\odot}$, 
$r_{t}<r_{s}$ and stars would be swallowed whole.) The numbers in this plot supersede the estimates in 32 which were based on the now-discredited Magorrian et al. 31] black hole masses. Disruption rates reach $\sim 10^{-3} \mathrm{yr}^{-1}$ in the faintest galaxies with verified black holes like M32. The dashed lines in that figure are the predicted rates in a singular isothermal sphere nucleus:

$$
\dot{N} \approx 2.5 \times 10^{-3} \mathrm{yr}^{-1}\left(\frac{\sigma}{100 \mathrm{~km} \mathrm{~s}^{-1}}\right)^{7 / 2}\left(\frac{M_{\bullet}}{10^{6} M_{\odot}}\right)^{-1} \propto M_{\bullet}^{-0.25}
$$

and in a nucleus with $\rho \propto r^{-3 / 2}$ :

$$
\dot{N} \approx 1.0 \times 10^{-3} \mathrm{yr}^{-1}\left(\frac{\sigma}{100 \mathrm{~km} \mathrm{~s}^{-1}}\right)^{21 / 5}\left(\frac{M_{\bullet}}{10^{6} M_{\odot}}\right)^{-4 / 5} \propto M_{\bullet}^{0.10}
$$

where the latter expressions in each case use the $M_{\bullet}-\sigma$ relation [41. The tidal flaring rate is probably nearly independent of $M_{\bullet}$ for $M_{\bullet} \lesssim 10^{6} M_{\odot}$, and if $\mathrm{dE}$ galaxies contain black holes, they would dominate the total flaring rate 60.

A fraction $25 \%-50 \%$ of a tidally disrupted star is expected to remain gravitationally bound to the black hole 2. If this is the case, and if disruption rates have remained nearly constant over $10^{10} \mathrm{yr}$, Figure [3 suggests that stellar consumption could be a major contributor to the growth of black holes with $M_{\bullet} \lesssim 10^{7} M_{\odot}$.

The feeding rates estimated above were based on a theory derived in the late 1970's to describe consumption rates of stars by massive black holes at the centers of globular clusters 3307. Globular clusters are many relaxation times old, and the assumption of a collisionally-relaxed steady state is built into the theory. For instance, the Cohn-Kulsrud [7] loss cone boundary conditions, used in Figure 3 are only valid in a collisionally relaxed system. But relaxation times in galactic nuclei can be very long and this fact requires a re-thinking of loss cone theory. Figure 4 shows histograms of three time scales relevant to feeding rates. The bottom panel is the relaxation time $T_{r}$ (eq. 3) evaluated at the black hole's influence radius $r_{h}$. $T_{r}$ exceeds $10^{10} \mathrm{yr}$ in most galaxies. The middle and upper panels show two time scales more directly related to the establishment of a steady-state loss cone. The time scale for scattering of low angular momentum stars into the loss cone is

$$
T_{\theta}(r) \approx \theta(r)^{2} T_{r}(r)
$$

where $\theta(r)$ is the scattering angle as seen from a star at radius $r$; the squareroot dependence of $\theta$ on $T_{\theta}$ reflects the fact that entry into the loss cone is a diffusive process. If the stellar phase space is initially well-populated by stars whose angular momenta are close to the critical value for capture $J_{l c}$, encounters will set up a steady state near the loss cone in the scattering time corresponding to $\theta=\theta_{l c} \approx\left(r_{t} / r\right)^{1 / 2}$, or

$$
T_{l c} \approx \frac{r_{t}}{r} T_{r} .
$$

Figure 4 shows that $T_{l c}$ is shorter than $10^{10} \mathrm{yr}$ in most galaxies. 


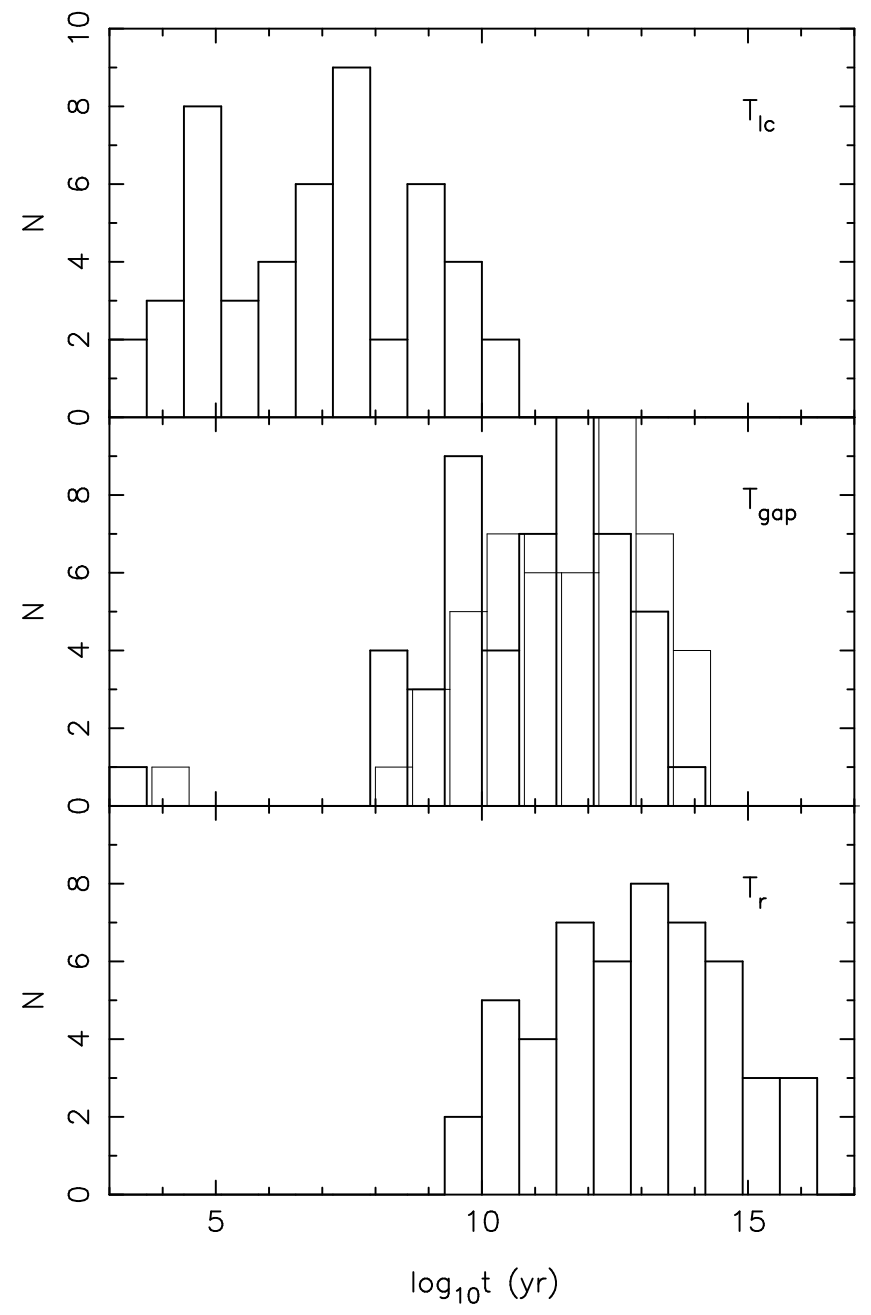

Fig. 4. Time scales $T_{r}, T_{l c}$ and $T_{g a p}$ defined in the text, for the sample of elliptical galaxies in Fig. 3. Thick/thin histograms of $T_{\text {gap }}$ are for $m_{2} / m_{1}=(0.1,1)$, where $m_{2} / m_{1}$ is the mass ratio of the binary black hole that preceded the current, single black hole.

But suppose that the loss cone was initially emptied of all stars with pericenters below some radius $r_{0} \gg r_{t}$; for instance, a binary black hole might have ejected such stars via the gravitational slingshot ( $\$ 4)$. Once the two black holes had coalesced, the time to diffusively fill this angular momentum gap would be

$$
T_{g a p} \approx \frac{r_{0}}{r} T_{r} \gg T_{l c}
$$


In the binary black hole model, most stars with pericenters

$$
r_{p} \lesssim K a_{h}=K \frac{G \mu}{4 \sigma^{2}}
$$

will have been ejected prior to the coalescence. Here $a_{h} \gg r_{t}$ is the semimajor axis of the binary when it first becomes "hard," $\mu$ is the reduced mass of the binary, and $K \approx 2$. The middle histogram in Figure 4 shows $T_{g a p}$ for two values of the binary mass ratio, $m_{2} / m_{1}=(0.1,1)$. In every galaxy,

$$
T_{l c} \ll T_{g a p}<T_{r} .
$$

Whereas $T_{l c}$ is generally shorter than $10^{10} \mathrm{yr}, T_{g a p}$ exceeds $10^{10} \mathrm{yr}$ in most galaxies and this is true for all "core" galaxies which show evidence of cusp destruction.

A more careful calculation 61], applying the time-dependent Fokker-Planck equation to the galaxies in Figure 3 shows that the time $t_{1 / 2}$ for the feeding rate to reach $1 / 2$ of its steady-state value after loss cone depletion by a binary black hole is approximately

$$
\frac{t_{1 / 2}}{10^{11} \mathrm{yr}} \approx \frac{\mu}{10^{7} M_{\bullet}} \approx 10 \frac{q}{(1+q)^{2}} \frac{M_{\bullet}}{10^{8} M_{\odot}}
$$

with $q=m_{2} / m_{1}$ the binary mass ratio. While this is a highly idealized model, it gives an indication of the time required for loss cone feeding to reach a steady state in galactic nuclei, and suggests that the tidal flaring rates in luminous "core" galaxies might be much lower than suggested by Figure 3

\section{Interaction of Binary Black Holes with Stars}

Binary supermassive black holes are inevitable consequences of galaxy mergers. Unless one of the black holes is very small, the two quickly form a bound pair at a separation $a \approx a_{h} \equiv G \mu / 4 \sigma^{2} \approx\left[\mu / 4\left(m_{1}+m_{2}\right)\right] r_{h}$ with $\mu$ the reduced mass. Subsequently the binary separation can decrease, but only if the binary is able to exchange angular momentum with stars or gas. (See 42 for a review of binarygas interactions.) Stars that pass within a distance $\sim 3 a$ of the binary undergo a complex 3-body interaction followed by ejection of the star at a velocity of order $\mu / m_{12}$ times the binary orbital velocity (the "gravitational slingshot" [56]). Each ejected star carries away energy and angular momentum, causing the semi-major axis, eccentricity, orientation, and center-of-mass velocity of the binary to change and the local density of stars to drop [25232446533637].

In principle, $N$-body studies can reveal both the long-term evolution of the binary as it interacts with stars, and the back-reaction of the binary on its stellar surroundings. But unless great care is taken, $N$-body simulations are likely to give misleading results. The reason is that time scales for two-body scattering of stars into the binary's loss cone are shorter by factors of $\sim N / 10^{11}$ in the simulations than in real galaxies, and the long-term evolution of the binary is 


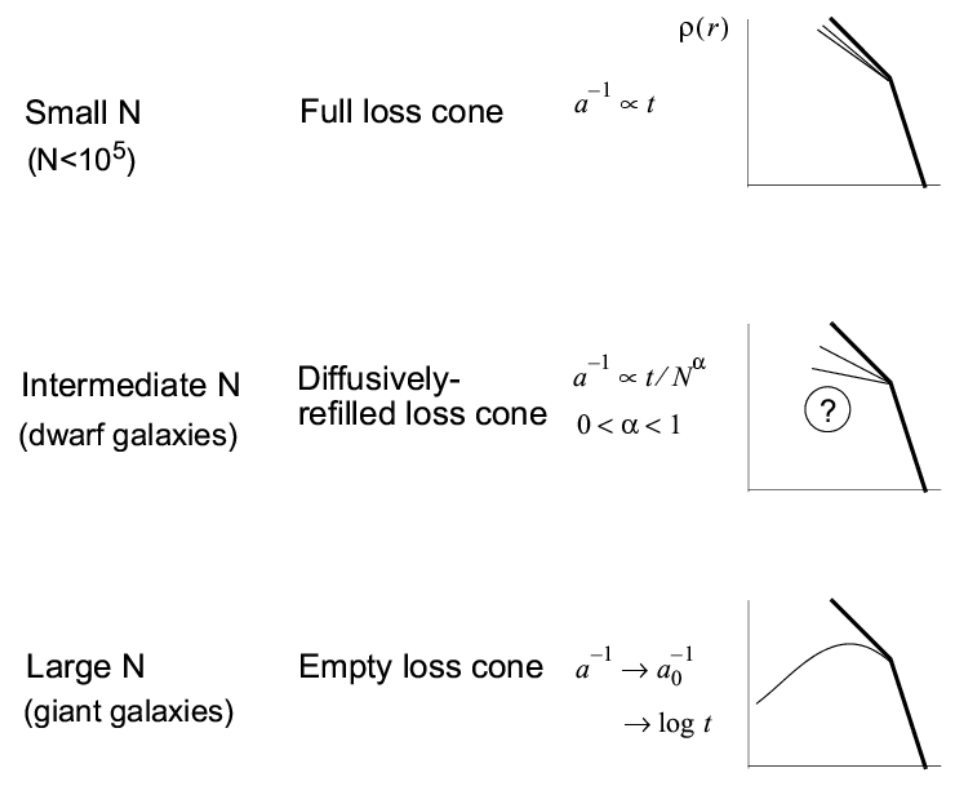

Fig. 5. Physical regimes for the decay of massive black hole binaries [48.

likely to be dominated by spurious loss cone refilling, wandering of the binary, and other noise-driven effects. Values of $N$ that are easily accessible to directsummation $N$-body simulation, $N \lesssim 10^{5}$, are so small that stars tend to be scattered into the binary's loss cone at a faster rate than they are kicked out by the gravitational slingshot 48. This is analogous to the "full loss cone" around a single black hole and it guarantees that the binary will never run out of stars.

$N$-body studies are most reliable when characterizing the early stages of binary formation and decay. Due to algorithmic limitations, most such studies 9343321635 have been based on galaxy models with unphysically large cores. The first simulations with realistically dense initial conditions 394743] showed that the stellar density around the binary drops very quickly after the binary first becomes hard, converting a $\rho \propto r^{-2}$ cusp to a shallower $\sim r^{-1}$ cusp.

But the long-term evolution of massive binaries in real galaxies, and the effect of binary evolution on nuclear structure, are not well understood. Figure [5 summarizes the likely regimes. For small $N$, of the order easily accessible to $N$-body simulations, collisionality is so high that the binary's loss cone remains full. The binary's binding energy increases linearly with time, $a^{-1} \propto t$, and the stellar density profile becomes gradually flatter. The same sort of evolution would occur in a triaxial nucleus where centrophilic orbits can continuously repopulate the loss cone [45]. At the other extreme, in giant galaxies, relaxation times are very long and almost no stars would be scattered into the binary's loss cone. The decay stalls, leaving a "hole" in the stellar density [67. A mechanism that might contribute to continued decay in this regime is re-ejection: stars ejected 


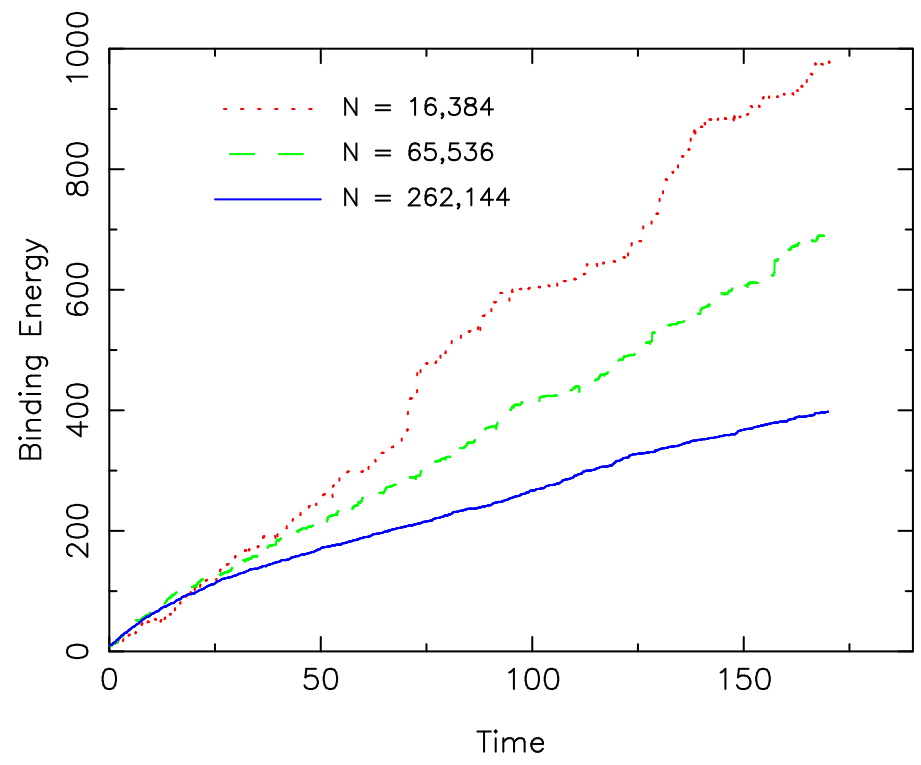

Fig. 6. $N$-dependence of the decay rate of a massive binary in $N$-body simulations [58. Each of the three runs started from the same initial state, an equal-mass binary with a binding energy of 10 . For small $N$, the long-term decay satisfies $a^{-1} \sim t$, the expected behavior in the full-loss-cone regime (Fig. 5). As $N$ increases, the lower degree of collisionality allows the loss cone around the binary to remain partially unfilled and decay slows.

with less than galactic escape velocity can continue to interact with the binary [48. The most interesting, and least-understood, regime is the intermediate one, corresponding to galaxies in which two-body scattering contributes substantially to the binary's decay without completely re-filling its loss cone 66. Reproducing this regime requires either $N$-body simulations with $N \gtrsim 10^{7}$, or a hybrid scheme that combines a Fokker-Planck integrator with a central binary. Figure 6 shows a step in this direction: $N$-body integrations for $N$ up to $0.25 \times 10^{6}$ using a GRAPE6 special-purpose computer, coupled with a chain regularization algorithm to follow close interactions of star and black hole particles [58. The $N$-dependence of the decay rate is clearly visible. Similar results were obtained [35] using a lower-accuracy $N$-body code. Increasing $N$ by an order of magnitude will soon be feasible and this will allow simulations in which the binary's evolution is dominated by slow diffusion into the loss cone, as in real galaxies.

\section{Brownian Motion}

Both single and binary black holes undergo a random walk in momentum space as their motion is perturbed by gravitational encounters with nearby stars. Simple encounter theory predicts that a massive black hole reaches a state of ki- 
netic energy equipartition with the stars, giving it a mean square velocity of $\left\langle V^{2}\right\rangle \approx 3\left(m / M_{\bullet}\right) \sigma^{2}$. This "gravitational Brownian motion" has been suggested as a mechanism for enhancing the supply of stars to a massive binary; indeed it has been asserted [546] that wandering of the binary's center of mass can itself guarantee a continued supply of stars.

While Brownian motion probably does affect the decay rate of binaries in the $N$-body simulations [4], it is doubtful that the effect is significant in real galaxies. The Brownian velocity of single black holes is found in $N$-body simulations to be 29

$$
\frac{1}{2} M_{\bullet}\left\langle V^{2}\right\rangle \approx \frac{3}{2} m \tilde{\sigma}^{2}
$$

where $\tilde{\sigma}^{2}$ is the $1 \mathrm{D}$, mean square stellar velocity within a region $r \lesssim 0.5 r_{h}$ around the black hole and $m$ is the stellar mass. Equation (12) holds in galaxy models with a wide range of nuclear density slopes, black hole masses and particle numbers up to $10^{6}$, the largest values so far used in direct-summation $N$-body simulations 8. In the case of the Milky Way black hole, equation (12) implies $V_{\mathrm{rms}} \approx 0.17 \mathrm{~km} \mathrm{~s}^{-1}$ (assuming $m=M_{\odot}$ ), a little larger than the "equipartition" value. The reason is that the black hole responds to perturbations from stars whose velocities have themselves been increased by the presence of the black hole 29 .

Brownian motion of a massive binary is larger than that of a single black hole, for two reasons [36]. (1) Stars are ejected superelastically from the binary, imparting a greater momentum to the binary than they would to a single black hole. (2) The dynamical friction force acting on the binary is less than that acting on a single particle due to the randomization of the ejection angles. The first factor dominates and produces an extra fractional contribution to $\left\langle V^{2}\right\rangle$ that is approximately $H /(32 \sqrt{2} \pi \ln \Lambda)$, with $H \approx 16$ the dimensionless hardening rate of the binary [53. Thus the Brownian motion of a binary is only slightly larger than that of a single black hole of the same mass. This prediction has been verified in $N$-body simulations 4735. The rms displacement of a binary from its otherwise central location would be very small in a real galaxy, probably even less than the separation between the two black holes.

The claim that wandering can guarantee a continued supply of stars to a binary is based on the following conceptual model [546]. As $N$ increases, the wandering first drops as expected from equation (12), but at sufficiently large $M_{\bullet} / m$ the binary empties its loss cone, producing a low-density core in which the binary is easily displaced. The amplitude of the binary's center-of-mass motion increases, allowing it to interact with a larger number of stars.

The motivation for this model was the complex $N$-dependence observed in a set of binary decay simulations 546: the decay rate first dropped with increasing $N$ until $N \approx 2 \times 10^{5}$, then remained constant when $N$ was doubled. But the postulated $N$-dependence of the wandering was never verified. Even if the interpretation in terms of wandering is correct, extrapolating the $N$-body results from $N \lesssim 10^{6}$ to $N \approx 10^{11}$ is problematic. Furthermore the galaxy models were Plummer spheres which have a much lower degree of concentration than 
real galaxies. and the wandering amplitudes inferred by these authors would be enormous if scaled to real galaxies, $\gtrsim 10^{2} \mathrm{pc}$, Extended simulations of binary hardening in realistic galaxy models with $N>10^{6}$ should soon settle this matter.

\section{Evidence for Cusp Destruction}

The structural parameters of elliptical galaxies display a smooth dependence on galaxy luminosity from $\mathrm{dE}$ galaxies up to the brighter ellipticals, $M_{V} \approx$ -20 18. Above this luminosity, galaxies generally have cores, regions near the center where the density falls below the inward extrapolation of the outer profiles (although the density almost always continues to rise inward). It is natural to attribute the cores to stellar ejection by a binary black hole and to use the "mass deficit" as a quantitative test of the binary black hole model (Fig. 7). In two studies 4955, pre-merger profiles for a sample of galaxies were constructed by extrapolating power laws, $\rho \propto r^{-\gamma_{0}}$, inward of the core radius. Milosavljevic et al. 49] found mean mass deficits of $\sim 1 M_{\bullet}$ for $\gamma_{0}=1.5$. More recently, Graham [19] fit the outer luminosity data to Sersic profiles and defined the mass deficit with respect to this template; mass deficits were found to be slightly larger, about twice $M_{\bullet}{ }^{1}$ These numbers are reasonable but it is difficult to say more given the difficulties cited above in interpreting $N$-body simulations of binary evolution.

An interesting unsolved problem is the effect of multiple mergers on the central density profiles of galaxies containing black holes. A simple argument suggests that the net effect, e.g. the core size, should increase both with the final black hole mass and with the number of merger events. Consider the merger of two galaxies with steep central cusps and black holes of mass $m_{1}$ and $m_{2}$; the result will be a galaxy with a shallower central profile and black hole of mass $m_{12}=m_{1}+m_{2}$. Now imagine that that same galaxy is produced by the merger of two galaxies whose central density cusps had already been destroyed by previous merger events. The final merger would lower the central density still more, producing a larger core for the same $m_{12}$. Testing this prediction via $N$-body simulations will be difficult for all the reasons discussed above, but it should be possible at least to test how the early evolution of binaries affects the central profiles of galaxies in multiple mergers.

The idea that cores grow cumulatively in mergers was used by Volonteri et al. 59] to predict the evolution of luminous and dark matter cores in galaxies. In their most extreme ("core preservation") model, the binaries were assumed to eject as much stellar mass as needed to shrink to a separation where gravitational radiation losses induce coalescence. The result, after $10^{10} \mathrm{yr}$, was cores with sizes of order $3 r_{h}$. While intriguing, this model lacks a firm foundation in the $N$-body simulations.

\footnotetext{
${ }^{1}$ Graham (2004) presents his mass deficits as significantly smaller than in the earlier study, but he bases his comparison exclusively on values of $M_{\text {def }}$ computed by Milosavljevic et al. using $\gamma_{0}=2$; the latter authors computed $M_{\text {def }}$ for a range of values $1.5 \leq \gamma_{0} \leq 2.0$.
} 


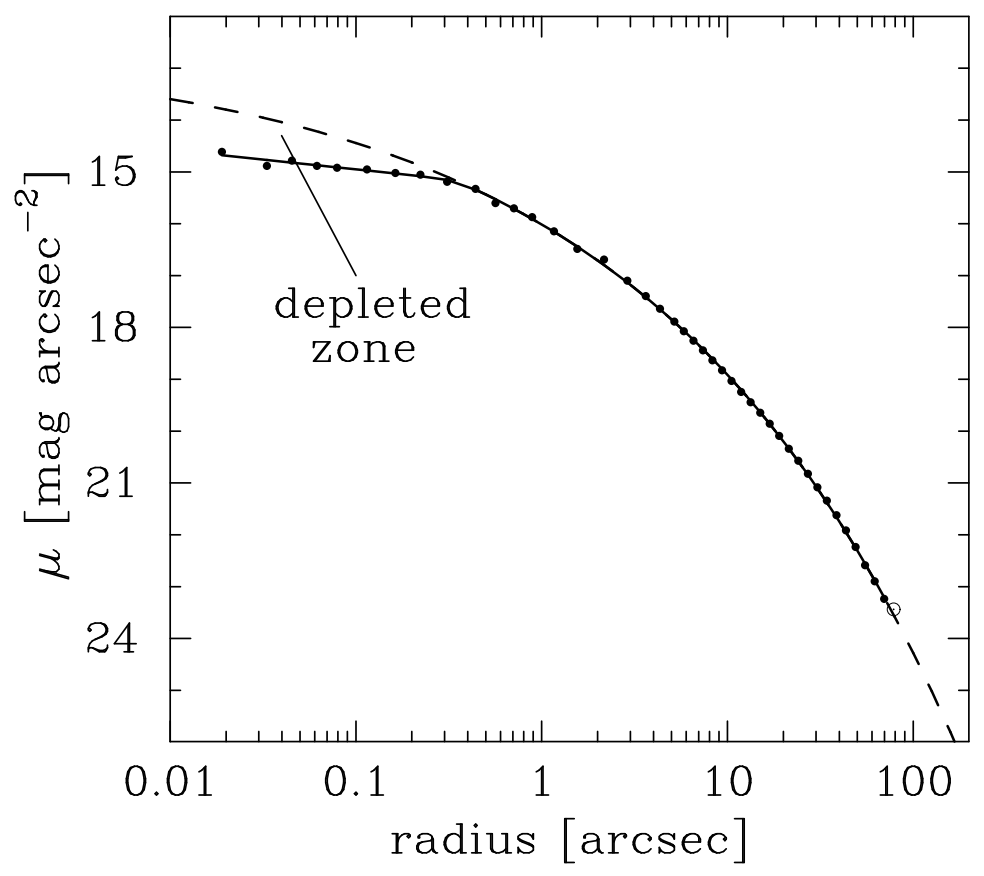

Fig. 7. Observed surface brightness profile of NGC 3348. The dashed line is a Sersic model fit to the large-radius data. The mass deficit is illustrated by the area designated "depleted zone" and the corresponding mass is roughly $3 \times 10^{8} M_{\odot}[19$.

In small dense galaxies, a destroyed cusp would be expected to re-form via the collisional mechanism discussed above, if the relaxation time at the core radius is less than the elapsed time since the merger. Alternatively, steep cusps may be due to star formation that occurred after the most recent merger.

There is one context in which formation of cores by black holes has been tested and shown to work. Globular clusters in the Large and Small Magellanic Clouds exhibit a range of ages and core sizes, and the mean core radius increases as the logarithm of the age [10. The observed age dependence is reproduced very well by a model in which the first population of stars leave behind $\sim 10 M_{\odot}$ remnants which sink to the center and displace the less massive stars before ejecting themselves via three-body interactions [40. This model may have some relevance to galactic nuclei if supermassive binaries stall long enough for third or fourth black holes to fall in.

\section{Black Hole Ejections}

If two black holes do manage to coalesce due to emission of gravitational radiation, linear momentum carried by the waves will impart a kick to the coalesced hole of amplitude $\sim 10^{-3} c$ [14]. The recoil velocity depends in a complicated 


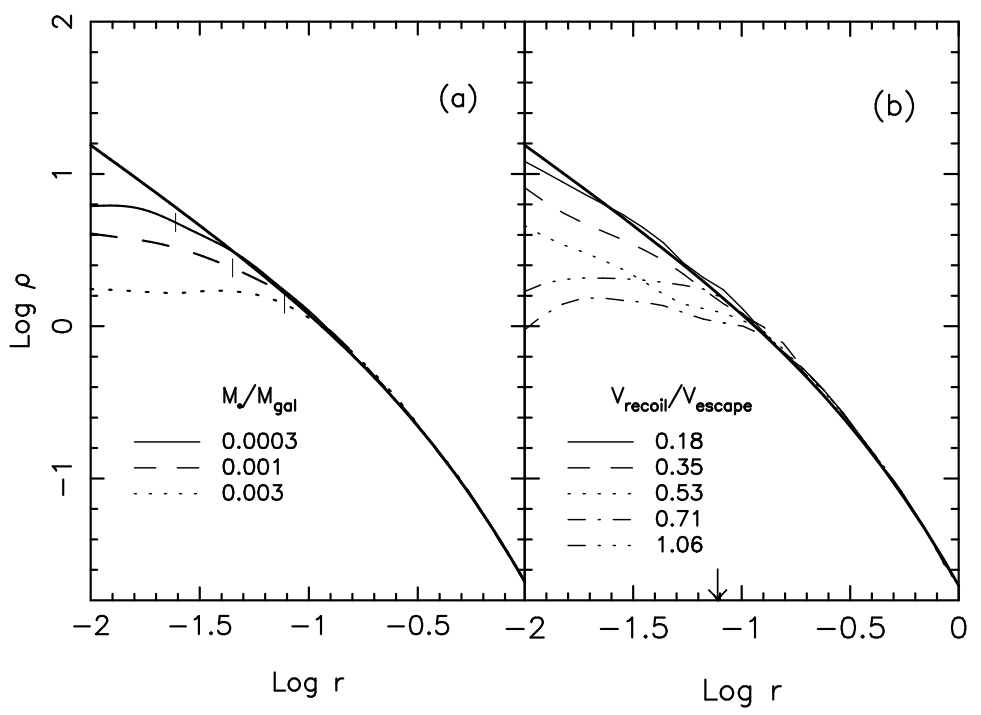

Fig. 8. Effect on the nuclear density profile of black hole ejection. The initial galaxy model (black line) has a $\rho \sim r^{-1}$ density cusp. (a) Impulsive removal of the black hole. Tick marks show the radius of the black hole's sphere of influence $r_{h}$ before ejection. A core forms with radius $\sim 2 r_{h}$. (b) Ejection at velocities less than escape velocity. The black hole has mass $0.003 M_{\text {gal }}$; the galaxy is initially spherical and the black hole's orbit remains nearly radial as it decays via dynamical friction. The arrow marks $r_{h}$.

way on the mass ratio and spins of the two black holes and on the spin orientations 12. At present only the mass ratio dependence is well understood: the recoil velocity peaks at $m_{2} / m_{1} \approx 0.38$ and falls to zero at $m_{2} / m_{1}=0$ or 1. As discussed by Hughes, Favata \& Holz in these proceedings, uncertainty about the strong-field behavior of the effect allows only plausible upper and lower limits to be placed on the magnitude of the kicks. However for moderately large spins and prograde capture, even the lower limits exceed $100 \mathrm{~km} \mathrm{~s}^{-1}$ for $0.2 \lesssim m_{2} / m_{1} \lesssim 0.6$, and the upper limit estimates reach $\sim 500 \mathrm{~km} \mathrm{~s}^{-1}$ for favorable mass ratios 44 .

An important possible consequence of the kicks is ejection of black holes from galaxies, particularly in the early universe when potential wells were shallow 44 2062. Whether or not a black hole is completely ejected, displacement of the black hole transfers energy to the nucleus and lowers its density within a region of size $\sim r_{h}$ (Fig. 8 ). Impulsive removal at $V_{\text {kick }} \gg V_{\text {escape }}$ produces a core of roughly constant density within a radius $\sim 2 r_{h}$ if the initial density profile is $\rho \propto r^{-1}$. The net effect is greater if $V_{\text {kick }} \lesssim V_{\text {escape }}$ since the black hole can return to the nucleus several times before settling down. For $V_{\text {kick }} \lesssim 0.25 V_{\text {escape }}$ the change in the density is negligible. Cores formed in this way have a similar size to those produced in the binary black hole model and complicate the interpretation of mass deficits. 


\section{References}

1. S. Aarseth: Pub. Astron. Soc. Pac. 111, 1333 (1999)

2. S. Ayal, M. Livio, T. Piran: Astrophys. J. 545, 772 (2000)

3. J. N. Bahcall, R. A. Wolf: Astrophys. J. 209, 214 (1976)

4. J. N. Bahcall, R. A. Wolf: Astrophys. J. 216, 883 (1977)

5. P. Chatterjee, L. Hernquist, A. Loeb: Astrophys. J. 572, 371 (2002)

6. P. Chatterjee, L. Hernquist, A. Loeb: Astrophys. J. 592, 32 (2003)

7. H. Cohn, R. Kulsrud: Astrophys. J. 226, 1087 (1978)

8. E. N. Dorband, M. Hemsendorf, D. Merritt: J. Comp. Phys. 185, 484 (2003)

9. T. Ebisuzaki, J. Makino, S. K. Okumura: Nature 354, 212 (1991)

10. R. Elson: Astrophys. J. Suppl. 76, 185 (1991)

11. S. M. Faber et al.: Astron. J. 114, 1771 (1997)

12. M. Favata, S. Hughes, D. Holz: Astrophys. J. Lett. 607, L5 (2004)

13. J. Frank, M. J. Rees: Mon. Not. R. Astron. Soc. 176, 633 (1976)

14. M. Fitchett: Mon. Not. R. Astron. Soc. 203, 1049 (1983)

15. R. Genzel et al.: Astrophys. J. 594, 812 (2003)

16. O. Gnedin, J. Primack: Phys. Rev. Lett. 93, 061302 (2004)

17. P. Gondolo, J. Silk: Phys. Rev. Lett. 83, 1719 (1999)

18. A. W. Graham, R. Guzman: Astron. J. 125, 2936 (2002)

19. A. W. Graham: Astrophys. J. 613, L33 (2004)

20. Z. Haiman: astro-ph/0404196 (2004)

21. M. Hemsendorf, S. Sigurdsson, R. Spurzem: Astrophys. J. 581, 1256 (2002)

22. J. G. Hills: Nature 254, 295 (1975)

23. J. G. Hills: Astron. J. 88, 1269 (1983)

24. J. G. Hills: Astron. J. 103, 1955 (1992)

25. J. G. Hills, L. W. Fullerton: Astron. J. 85, 1281 (1980)

26. D. Hooper, I. Perez, J. Silk, F. Ferrer, S. Sarkar: J. Cosmol. Astropart. Phys. 9, 2 (2004)

27. A. S. Ilyin, K. P. Zybin, A. V. Gurevich: astro-ph/0306490 (2003)

28. T. R. Lauer et al.: Astron. J. 116, 2263 (1998)

29. F. Laun, D. Merritt: astro-ph/0408029 (2004)

30. A. P. Lightman, S. L. Shapiro: Astrophys. J. 211, 244 (1977)

31. J. Magorrian et al.: Astron. J. 115, 2285 (1998)

32. J. Magorrian, S. Tremaine: Mon. Not. R. Astron. Soc. 309, 447 (1999)

33. J. Makino: Astrophys. J. 478, 58 (1997)

34. J. Makino, T. Fukushige, S. K. Okumura, T. Ebisuzaki: Pub. Astron. Soc. J. 45, 303 (1993)

35. J. Makino, Y. Funato:Astrophys. J. 602, 93 (2004)

36. D. Merritt: Astrophys. J. 556, 245 (2001)

37. D. Merritt: Astrophys. J. 568, 998 (2002)

38. D. Merritt: Phys. Rev. Lett. 92, 201304 (2004)

39. D. Merritt, F. Cruz: Astrophys. J. Lett. 551, L41 (2001)

40. D. Merritt, S. Piatek, S. Portegies Zsart, M. Hemsendorf: Astrophys. J. Lett. 608, L25 (2004)

41. D. Merritt, L. Ferrarese: 'Relation of Black Holes to Bulges'. In: The Central Kiloparsec of Starbursts and AGN, ASP Conf. Ser. Vol. 249, ed. by J. H. Knapen et al. (ASP, San Francisco 2001) pp. 335-362

42. D. Merritt, M. Milosavljevic: Massive Binary Black Hole Evolution, Living Reviews in Relativity (2004) 
43. D. Merritt, M. Milosavljevic, L. Verde, R. Jimenez: Phys. Rev. Letts. 88, 191301 (2002)

44. D. Merritt, M. Milosavljevic, M. Favata, S. Hughes, D. Holz: Astrophys. J. Lett. 607, L9 (2004)

45. D. Merritt, M. Y. Poon: Astrophys. J. 606, 788 (2004)

46. S. Mikkola, M. J. Valtonen: Mon. Not. R. Astron. Soc. 259, 115 (1992)

47. M. Milosavljevic, D. Merritt: Astrophys. J. 563, 34 (2001)

48. M. Milosavljevic, D. Merritt: Astrophys. J. 596, 860 (2003)

49. M. Milosavljevic, D. Merritt, A. Rest, F. C. van den Bosch: Mon. Not. R. Astron. Soc. 331, L51 (2002)

50. B. W. Murphy, H. N. Cohn, R. H. Durisen: Astrophys. J. 370, 60 (1991)

51. P. J. E. Peebles: Gen. Rel. Grav. 3, 61 (1972)

52. M. Preto, D. Merritt, R. Spurzem: Astrophys. J. 613, 000 (2004)

53. G. D. Quinlan: New Astron. 1, 35 (1996)

54. G. D. Quinlan, L, Hernquist: New Astron. 2, 533 (1997)

55. S. Ravindranath, L. C. Ho, A. V. Filippenko: Astrophys. J. 566801 (2002)

56. W. C. Saslaw, M. J. Valtonen, S. J. Aarseth: Astrophys. J. 190, 253 (1974)

57. S. L. Shapiro, A. P. Lightman: Nature 262, 743 (1976)

58. A. Szell, D. Merritt: in preparation (2004)

59. M. Volonteri, P. Madau, F. Haardt: Astrophys. J. 593, 661 (2003)

60. J. Wang, D. Merritt: Astrophys. J. 600, 149 (2004)

61. J. Wang, D. Merritt: in preparation

62. J. Yoo, J. Miralda-Escude: astro-ph/0406217 (2004)

63. P. J. Young: Astrophys. J. 215, 36 (1977)

64. P. J. Young: Astron. J. 242, 1232 (1980)

65. P. J. Young, G. R. Shields, J. C. Wheeler: Astrophys. J. 212, 367 (1977)

66. Q. Yu: Mon. Not. R. Astron. Soc. 331, 935 (2002)

67. C. Zier, P. L. Biermann: Astron. Astrophys. 377, 23 (2001)

68. H. Zhao, M. G. Haehnelt, M. J. Rees: New Astron. 7, 385 (2002) 